\title{
LA VULNERABILIDAD URBANA Y SU CARACTERIZACIÓN SOCIO-ESPACIAL
}

Ochoa-Ramírez, José Alberto; Guzmán-Ramírez, Alejandro

LA VULNERABILIDAD URBANA Y SU CARACTERIZACIÓN SOCIO-ESPACIAL

Revista Legado de Arquitectura y Diseño, vol. 15, núm. 27, 2020

Universidad Autónoma del Estado de México, México

Disponible en: http://www.redalyc.org/articulo.oa?id=477963263004

Esta obra está bajo una Licencia Creative Commons Atribución-NoComercial-SinDerivar 4.0 Internacional. 


\title{
LA VULNERABILIDAD URBANA Y SU CARACTERIZACIÓN SOCIO- ESPACIAL
}

\author{
THE URBAN VULNERABILITY AND ITS SOCIO- \\ SPACE CHARACTERIZATION
}

José Alberto Ochoa-Ramírez jose.ochoa@ugto.mx

Universidad de Guanajuato, México

Alejandro Guzmán-Ramírez alejandro.guzman@ugto.mx

Universidad de Guanajuato, México

Revista Legado de Arquitectura y Diseño, vol. 15 , núm. 27,2020

Universidad Autónoma del Estado de México, México

Recepción: 17 Octubre 2019 Aprobación: 02 Diciembre 2019

Redalyc: http://www.redalyc.org/ articulo.oa?id $=477963263004$
Resumen: La vulnerabilidad se define como un estado de elevada exposición a determinados riesgos e incertidumbres, combinado con una capacidad disminuida para protegerse o defenderse de ellos y hacer frente a sus consecuencias negativas.

A partir de la revisión teórico-conceptual sobre la vulnerabilidad, se implementa una metodología de análisis de indicadores sobre la vulnerabilidad socio-demográfica, la vulnerabilidad socio-económica y la vulnerabilidad socio-espacial, que contribuyan a delimitar áreas territoriales para identificar aspectos clave de vulnerabilidad urbana presentes en la ciudad de León, Guanajuato, México.

Palabras clave: desigualdad social, marginación urbana, vulnerabilidad urbana.

Abstract: The vulnerability can be defined as a state of high exposure to certain risks and uncertainties, combined with a decreased ability to protect or defend themselves against them and cope with their negative consequences.

Based on the theoretical-conceptual review of vulnerability and the implementation of a methodology for the analysis of indicators on socio-demographic vulnerability, socioeconomic vulnerability and socio-spatial vulnerability, which contribute delimit territorial areas to identify key aspects of urban vulnerability present in the city of Leon, Guanajuato, Mexico.

Keywords: social inequality, urban marginalization, urban vulnerability.

\section{INTRODUCCIÓN}

Esta investigación contribuirá al conocimiento sobre los diversos procesos y factores que puedan identificar los sectores urbanos con vulnerabilidad urbana, será una base para que desde el ámbito académico se pueda proveer información científica y fundamentada que puede ser útil para concientizar y orientar, tanto las intervenciones urbano-arquitectónicas como las de índole social.

El presente estudio sobre la vulnerabilidad urbana se estructuró en cuatro etapas basadas en los siguientes objetivos y alcances:

- Análisis de los perfiles teóricos empleados en la conceptualización de la vulnerabilidad urbana; que nos permita, identificar los indicadores más utilizados para su definición.

- Selección de los indicadores, su clasificación y discriminación que permitan acotar la vulnerabilidad urbana. 
- Delimitación de las zonas vulnerables en la ciudad de León, Guanajuato, con base al contraste de indicadores seleccionados y la información geo-estadística existente.

- Descripción de la caracterización socio-espacial de los polígonos vulnerables delimitados.

\section{ANTECEDENTES TEÓRICOS}

En términos generales, el concepto de vulnerabilidad hace referencia a dos cuestiones (ONU, 2003):

a) El incremento de las amenazas y los riesgos que afectan a las personas/sociedades/grupos sociales/estados.

b) El debilitamiento de los mecanismos para afrontar dichos riesgos y amenazas.

Así mismo, se puede plantear el estudio de la vulnerabilidad a partir de tres ejes básicos (Pizarro, 2001; Rodríguez, 2001):

La vulnerabilidad socio-demográfica: Refiere a grupos sociales vulnerables, a dinámicas demográficas que condicionen el desarrollo de la población en un territorio; pero sobre todo con los niveles de bienestar de la población en educación, salud y cobertura de servicios.

La vulnerabilidad socio-económica: Tiene que ver con la capacidad económica de los distintos estratos de la población; también refiere a la base de recursos -activos y pasivos- que hacen a una ciudad capaz de atender por sí misma las emergencias y recuperarse, y sobre todo con la distribución de la riqueza en la sociedad.

La vulnerabilidad espacial: Los soportes físicos que acogen la vida de los ciudadanos son muy importantes, puesto que sus malas condiciones no permitirán un desarrollo satisfactorio de la vida cotidiana, puesto que es una dimensión básica de la condición humana la adecuación del hábitat a las necesidades de la población.

Otro factor a considerar sobre la vulnerabilidad, son

LAS PERCEPCIONES SUBJETIVAS DE LA POBLACIÓN,

YA QUE LA DIMENSIÓN CULTURAL, JUNTO CON LA DIMENSIÓN AMBIENTAL

Y LA IDEOLÓGICA

forman un contexto en el que hábitos, costumbres, creencias, valores

IMPLICAN INFLUENCIAS DETERMINANTES EN UN ESTADO DE VULNERABILIDAD SOCIAL.

Otro factor a considerar sobre la vulnerabilidad, son las percepciones subjetivas de la población, ya que la dimensión cultural, junto con la dimensión ambiental y la ideológica forman un contexto en el que hábitos, costumbres, creencias, valores implican influencias determinantes en un estado de vulnerabilidad social.

Puede entenderse por vulnerabilidad urbana "como aquel proceso de malestar en las ciudades producido por la combinación de múltiples dimensiones de desventaja, en el que toda esperanza de movilidad social ascendente, de superación de su condición social de exclusión o próxima 
a ella, es contemplada como extremadamente difícil de alcanzar. Por el contrario, conlleva una percepción de inseguridad y miedo a la posibilidad de una movilidad social descendente, de empeoramiento de sus actuales condiciones de vida" (ONU, 2003).

En este sentido, la vulnerabilidad urbana hace referencia a tres aspectos básicos (Arriba, 2008):

a) Condiciones de riesgo ambiental que sufren ciertos sectores de la población al estar asentados en zonas no seguras por sus condiciones topográficas o aéreas propensas a desastres naturales; o bien aquellas que se ubican en aéreas prohibidas por considerarse zonas peligrosas por la actividad humana.

b) Condiciones de habitabilidad que hacen referencia, tanto a las viviendas como al entorno urbano, en ese sentido, un hábitat degradado, tanto si afecta a las edificaciones como al espacio urbano, son expresiones de condiciones desfavorables que pueden propiciar la aparición de sentimientos de vulnerabilidad.

c) Cobertura de servicios, infraestructura y equipamiento, constituye otra expresión de la vulnerabilidad urbana, aquellos territorios que no reúnen condiciones dignas de habitabilidad, ya sea por el estado de deterioro de las edificaciones, por disponer de una superficie insuficiente o bien por la falta de servicios básicos.

\section{MATERIALES Y MÉTODOS}

Se plantean tres enfoques de estudio sobre la vulnerabilidad basados en el análisis de distintos conceptos encontrados que comprenden la vulnerabilidad socio-demográfica (a), la vulnerabilidad socio-económica (b) y la vulnerabilidad socio-espacial (c); identificando como sus indicadores principales los siguientes:

a) Desarrollo humano y desigualdad social

b) Rezago social y marginación

c) Consolidación urbana y vulnerabilidad

\section{a) Desarrollo humano y desigualdad social}

Tanto la pobreza y como la desigualdad, son fenómenos muy complejos, cuyo análisis no puede restringirse a la dimensión económica; requieren adoptar una perspectiva multidimensional utilizando indicadores como: educación, salud o a los bienes de la ciudad, cuyo acceso, calidad y distribución suele ser muy inequitativo.

Por ello conviene definir los límites conceptuales que existen entre la pobreza y la desigualdad urbana, ya que son conceptos que, aunque suelen usarse indistintamente y están interrelacionados son sustancialmente diferentes. La pobreza es un complejo proceso de privación y escasez de recursos económicos sociales, culturales, institucionales, políticos y 
también territoriales que afecta a los sectores populares y que está asociado principalmente a las condiciones de inserción que prevalecen en el mercado de trabajo: inestabilidad, informalidad, bajos salarios, precariedad laboral (Boltvinik, 1999).

La desigualdad, en cambio, es un concepto relacional, de diferencias y dispersión de la distribución del ingreso y de los recursos en una sociedad. Es claro entonces que la desigualdad está fuertemente relacionada con la pobreza, pero también con la riqueza; cuanto más desigual es la distribución del ingreso mayor será el porcentaje de la población en situación de pobreza, pero a ello se agrega que en las grandes ciudades es donde las formas diferenciadas de acceso y calidad de la vivienda y los bienes y servicios colectivos -agua, drenaje, equipamientos, espacios públicos o transporte de calidad-son indicadores inequívocos de grandes desigualdades que existen en el territorio (Corredor, 2008).

\section{Desigualdad social}

Esta metodología es desarrollada por la unión europea y consiste generar un instrumento para aproximarnos al análisis de la desigualdad social en las ciudades mediante el estudio de las necesidades sociales, la pobreza o la calidad de vida (ETSAM, 2014).

En dimensiones, tales como (tabla 1):

- Demográficas. Características demográficas de los que residen en la ciudad.

- Mercado de trabajo. En este apartado se presenta la situación laboral de la población, así como las características y evolución del mercado de trabajo.

- Educativas. Este indicador informa de la situación educativa en los barrios, y de las características propias de la población y su posible relación con otros problemas como desempleo.

- Salud. Las condiciones sanitarias de la población y acceso a servicios de salud.

- Vivienda. La tipificación de la vivienda relacionada con los colectivos que tienen menos ingresos económicos; las viviendas públicas desarrolladas a través de programas sociales y la cobertura de equipamientos básicos. 
Tabla 1. Indicadores de desigualdad social.

\begin{tabular}{|l|l|l|l|l|}
\hline Indicadores de desigualdad social & $\begin{array}{l}\text { Nivel de } \\
\text { instrucción }\end{array}$ & Salud & Vivienda \\
\hline Demografia & Población activa & Analfabetismo & $\begin{array}{l}\text { Esperanza de } \\
\text { vida al nacer }\end{array}$ & $\begin{array}{l}\text { Viviendas de } \\
\text { carácter social }\end{array}$ \\
\hline $\begin{array}{l}\text { Dependencia } \\
\text { demográfica }\end{array}$ & $\begin{array}{l}\text { Tasa de } \\
\text { desempleo }\end{array}$ & $\begin{array}{l}\text { Tasa de } \\
\text { instrucción } \\
\text { insuficiente }\end{array}$ & Mortalidad & $\begin{array}{l}\text { Viviendas sin } \\
\text { equipamientos } \\
\text { básicos }\end{array}$ \\
\hline $\begin{array}{l}\text { Población } \\
\text { infantil }\end{array}$ & $\begin{array}{l}\text { Tasa de } \\
\text { titulados } \\
\text { universitarios }\end{array}$ & & $\begin{array}{l}\text { Número de } \\
\text { personas por } \\
\text { vivienda }\end{array}$ \\
\hline $\begin{array}{l}\text { Población } \\
\text { mayor de } 65 \\
\text { años }\end{array}$ & ocupación & & & \\
\hline $\begin{array}{l}\text { Índice de } \\
\text { envejecimiento }\end{array}$ & & & & \\
\hline
\end{tabular}

Fuente: Elaboración propia con base en ETSAM (2014).

\section{Desarrollo Humano}

El Desarrollo Humano es un proceso mediante el cual se busca la ampliación de las oportunidades para las personas, aumentando sus derechos y capacidades. Este proceso incluye varios aspectos de la interacción humana como la participación, la equidad de género, la seguridad, la sostenibilidad, las garantías de los derechos humanos y otros que son reconocidos por la gente como necesarias para ser creativos y vivir en paz.

El desarrollo consiste en la ampliación de las opciones que las personas tienen para vivir de acuerdo con sus valores y aspiraciones. Por lo que se definen tres indicadores principales: una vida longeva y sana, medida por las esperanzas de vida al nacer; el conocimiento, medido por la tasa de analfabetismo adulto y la tasa de matrícula total combinada de primaria, secundaria y terciaria; y un nivel de vida decente, medido por el Producto Interno Bruto per cápita (PNUD, 2016).

$\mathrm{Si}$ no se poseen estas oportunidades esenciales, muchas otras alternativas continuarán siendo inaccesibles; pero el desarrollo humano no termina allí; otras oportunidades, altamente valoradas por muchas personas, van desde la libertad política, económica y social, hasta la posibilidad de ser creativo y productivo, respetarse a sí mismo y disfrutar de la garantía de Derechos Humanos (tabla 2). 
Tabla 2. Indicadores de Desarrollo Humano.

\begin{tabular}{|c|c|c|c|c|}
\hline \multicolumn{5}{|c|}{ Indicadores de Desarrollo Humano } \\
\hline Participación & Protección & Entendimiento & Subsistencia & Identidad \\
\hline $\begin{array}{l}\text { Igualdad de } \\
\text { oportunidades }\end{array}$ & $\begin{array}{l}\text { Sistema de } \\
\text { salud }\end{array}$ & Educación & Alimentación & $\begin{array}{l}\text { Valores } \\
\text { culturales } \\
\text { locales }\end{array}$ \\
\hline Equidad & Asistencia legal & $\begin{array}{l}\text { Desarrollo } \\
\text { integral }\end{array}$ & Ingreso & $\begin{array}{l}\text { Valores } \\
\text { Culturales } \\
\text { regionales }\end{array}$ \\
\hline \multirow[t]{2}{*}{$\begin{array}{l}\text { Participación } \\
\text { social }\end{array}$} & & & Abrigo & \\
\hline & & & Vivienda & \\
\hline
\end{tabular}

Fuente: Elaboración propia con base en PNUD (2016).

\section{b) Rezago social y marginación}

La marginación y el rezago se asocian a la carencia de oportunidades sociales y a la ausencia de capacidades para adquirirlas o generarlas, pero también a privaciones e inaccesibilidad a bienes y servicios fundamentales para el bienestar. En consecuencia, las comunidades marginadas enfrentan escenarios de elevada vulnerabilidad social, cuya mitigación escapa del control personal o familiar, pues esas situaciones no son resultado de elecciones individuales, sino de un modelo productivo que no brinda a todos las mismas oportunidades (Cortez, 2002).

Las desventajas ocasionadas por la marginación son acumulables, configurando escenarios cada vez más desfavorables. Mientras que la medición del rezago social incorpora indicadores que ayudan a conocer el nivel de desigualdad económica y social de la población; a través de variables de educación, de acceso a servicios de salud, de servicios básicos en la vivienda, de calidad y espacios en la misma, y de activos en el hogar (Feres, Mancero, 2001).

\section{Marginación}

La función central de los indicadores de marginación consiste en ser un punto de referencia para la aplicación de estrategias y políticas públicas de desarrollo social; la marginación urbana permite dar cuenta del fenómeno estructural de la distribución de los bienes y servicios de los cuales debe gozar toda la población (CONAPO, 2015).

De alguna forma también nos habla de la desigualdad social, ya que los bienes del desarrollo no llegan de la misma forma a toda la población. Así, el concepto de marginación se aplica a través de los componentes de educación, salud, condiciones de la vivienda, ingreso y bienes en la vivienda (tabla 3 ). 
Tabla 3. Indicadores de marginación.

\begin{tabular}{|c|c|c|c|c|}
\hline \multicolumn{5}{|c|}{ Indicadores de marginación } \\
\hline Economia & Educación & Salud & Vivienda & Bienes \\
\hline $\begin{array}{l}\text { Población } \\
\text { ocupada }\end{array}$ & Analfabetismo & $\begin{array}{l}\text { Sistema y } \\
\text { servicio de } \\
\text { salud }\end{array}$ & $\begin{array}{l}\text { Calidad } \\
\text { constructiva } \\
\text { (viviendas con } \\
\text { piso de tierra) }\end{array}$ & $\begin{array}{l}\text { Posesión de } \\
\text { activos } \\
\text { (refrigerador o } \\
\text { bienes de } \\
\text { consumo } \\
\text { duradero) }\end{array}$ \\
\hline \multirow[t]{2}{*}{$\begin{array}{l}\text { Nivel de ingreso } \\
\text { (menos de dos } \\
\text { salarios } \\
\text { mínimos) }\end{array}$} & $\begin{array}{l}\text { Nivel educativo (con } \\
\text { primaria, con } \\
\text { secundaria } \\
\text { incompleta/completa) }\end{array}$ & $\begin{array}{l}\text { Tasa de } \\
\text { nacimiento, } \\
\text { mortalidad, } \\
\text { esperanza de } \\
\text { vida al nacer }\end{array}$ & $\begin{array}{l}\text { Servicios } \\
\text { básicos } \\
\text { (drenaje, agua } \\
\text { entubada, } \\
\text { electricidad) }\end{array}$ & \\
\hline & & & $\begin{array}{l}\text { Nivel de } \\
\text { hacinamiento }\end{array}$ & \\
\hline
\end{tabular}

Fuente: Elaboración propia con base en CONAPO (2015).

\section{Rezago social}

La medición del rezago social incorpora indicadores que ayudan a conocer el nivel de desigualdad económica y social de la población, lo que permite aproximarse al nivel de equidad y solidaridad que existe en una sociedad.

A través de los siguientes indicadores (CONEVAL, 2010):

Coeficiente de Gini: mide la desigualdad económica de una sociedad, mediante la exploración del nivel de concentración que existe en la distribución de los ingresos entre la población.

Razón de ingreso: Permite conocer la brecha que existe entre los ingresos de las personas en pobreza extrema respecto al de las personas no pobres y no vulnerables.

Grado de polarización social: mide las diferencias que existen entre las condiciones de vida de la población que vive en un mismo lugar (tabla 4).

Tabla 4. Indicadores de rezago social.

\begin{tabular}{|l|l|l|}
\hline \multicolumn{2}{|l|}{ Indicadores de rezago social } & \\
\hline Coeficiente de Gini & Razón de ingreso & Polarización social \\
\hline $\begin{array}{l}\text { Desigualdad en la } \\
\text { distribución de ingreso }\end{array}$ & $\begin{array}{l}\text { Personas en pobreza } \\
\text { extrema }\end{array}$ & $\begin{array}{l}\text { Vivienda - ingreso - nivel } \\
\text { educativo }\end{array}$ \\
\hline $\begin{array}{l}\text { Equidad en la distribución } \\
\text { de ingreso }\end{array}$ & $\begin{array}{l}\text { Personas no pobres y no } \\
\text { vulnerables }\end{array}$ & $\begin{array}{l}\text { Polo izquierdo (alta } \\
\text { marginación) Polo derecho } \\
\text { (baja marginación) }\end{array}$ \\
\hline
\end{tabular}

Fuente: Elaboración propia con base en CONEVAL (2010).

\section{c) Consolidación urbana y vulnerabilidad}

Es necesario explorar cuáles son los factores espaciales que generan la vulnerabilidad, en el entendido de que ésta no se deriva exclusivamente de las condiciones socioeconómicas individuales o de los hogares. Además, cobran relevancia otras características del espacio geográfico que inciden 
directamente en la existencia de satisfactores y en las probabilidades de acceder a ellos. Esto implica que aparte de estudiar la distribución de características y recursos de individuos, es necesario determinar los atributos explícitamente espaciales de las unidades geográficas donde aquellos se localizan.

En particular, a los recursos e infraestructuras que forman la base material de los servicios de salud, educativos, del transporte y de uso de los espacios públicos, deportivos y culturales. La razón para enfocarse en ellos es su influencia determinante sobre la accesibilidad a bienes y servicios, así como sobre los perfiles de consumo, el empleo y el ingreso (Palacio-Prieto et al., 2004).

\section{Consolidación urbana}

La consolidación urbana se evalúa por dos factores principales: a) el nivel de urbanización caracterizado por la cobertura de servicios públicos, por equipamiento básico, calidad en la infraestructura existente y densificación urbana; b) la conectividad con centros urbanos y la accesibilidad a bienes y servicios, que incluye la infraestructura necesaria para soportar la diversidad de actividades productivas (Delgado, 2007).

Las variables que integran la consolidación urbana (Suárez 2012) son:

1) Aspectos económicos;

2) Aspectos urbanos;

3) Aspectos sociales (tabla 5).

Tabla 5. Indicadores de consolidación urbana.

\begin{tabular}{|l|l|l|}
\hline \multicolumn{2}{|l|}{ Indicadores de consolidación urbana } \\
\hline Aspectos económicos & Aspectos urbanos & Aspectos sociales \\
\hline $\begin{array}{l}\text { Población Económicamente } \\
\text { Activa PEA }\end{array}$ & Nivel de urbanización & Inmigración \\
\hline $\begin{array}{l}\text { Alta y baja calificación de } \\
\text { empleo }\end{array}$ & $\begin{array}{l}\text { Distancia al centro } \\
\text { metropolitano más cercano }\end{array}$ & Movilidad social \\
\hline
\end{tabular}

Fuente: Elaboración propia con base en Suarez (2012).

\section{Vulnerabilidad}

La vulnerabilidad puede definirse como un estado de exposición a determinados riesgos e incertidumbres, combinado con una capacidad disminuida para protegerse o defenderse de ellos y hacer frente a sus consecuencias negativas (MFE, 2010).

Así pues, la vulnerabilidad de un territorio tiene que ver tanto con condiciones objetivas como relativas que la afectan: por un lado, está constituida por condiciones de desfavorecimiento social, de desventajas estructurales de una población para desarrollar proyectos vitales en contextos de seguridad y confianza; por el otro, la vulnerabilidad es también un estado psicosocial que afecta a la percepción que los 
ciudadanos tienen del territorio en donde viven y de sus propias condiciones sociales (tabla 6).

Tabla 6. Indicadores de vulnerabilidad.

\begin{tabular}{|c|c|c|c|}
\hline \multicolumn{4}{|c|}{ Indicadores de Vulnerabilidad } \\
\hline Sociodemográfica & Socioeconómica & Residencial & Subjetiva \\
\hline $\begin{array}{l}\text { Población de } 75 \\
\text { años o más }\end{array}$ & $\begin{array}{l}\text { Población activa } \\
\text { desempleada }\end{array}$ & $\begin{array}{l}\text { Vivienda con menos } \\
\text { de } 30 \mathrm{~m}^{2}\end{array}$ & $\begin{array}{l}\text { Población con } \\
\text { contaminación en su } \\
\text { entorno }\end{array}$ \\
\hline $\begin{array}{l}\text { Hogares } \\
\text { unipersonales de } \\
\text { mayores de } 64 \text { años }\end{array}$ & $\begin{array}{l}\text { Ocupados } \\
\text { eventuales } \\
\text { ocupados no } \\
\text { Cualificados }\end{array}$ & $\begin{array}{l}\text { Población en } \\
\text { viviendas sin } \\
\text { servicios básicos }\end{array}$ & $\begin{array}{l}\text { Población con } \\
\text { problemas de } \\
\text { escasez de áreas } \\
\text { verdes }\end{array}$ \\
\hline $\begin{array}{l}\text { Hogares con un } \\
\text { adulto y un menor } \\
\text { más }\end{array}$ & $\begin{array}{l}\text { Población sin } \\
\text { estudios }\end{array}$ & $\begin{array}{l}\text { Viviendas en } \\
\text { edificios en mal } \\
\text { estado de } \\
\text { conservación }\end{array}$ & $\begin{array}{l}\text { Población con } \\
\text { problemas de } \\
\text { inseguridad en su } \\
\text { entorno }\end{array}$ \\
\hline
\end{tabular}

Fuente: Elaboración propia con base en MFE (2010).

\section{RESULTADOS}

Se elaboró un diagnóstico de estudio en la ciudad de León, Guanajuato, México con base en:

1. Análisis socio-demográfico. Análisis de las condiciones de rezago de grupos de población o perfiles sociales reconocidos como vulnerables.

Con base al análisis e interpretación de los datos obtenidos de CONEVAL (2014), en el municipio de León, las personas que se encuentran vulnerables por carencias sociales es de 446754 , lo que equivale al $31.1 \%$ de la población; el número de personas que se encuentran vulnerables por carencia en rezago educativo es de 330.786 , lo que equivale al $20.8 \%$ de la población; el número de personas que se encuentran vulnerables por carencia de servicios de salud es de 442068 , lo que equivale al $27.8 \%$ de la población; el número de personas que se encuentran vulnerables por carencia de seguridad social es de 843651 , lo que equivale al $53.1 \%$ de la población; el número de personas que se encuentran vulnerables por carencia de calidad y espacios en la vivienda es de 110 489, lo que equivale al 7.0\% de la población; el número de personas que se encuentran en carencia de servicios básicos en la vivienda es de 189 265, lo que equivale al 11.9\% de la población; mientras que el número de personas que se encuentran vulnerables por carencia de acceso a la alimentación es de 315 938, lo que equivale al 19.9\% de la población (figura 1). 


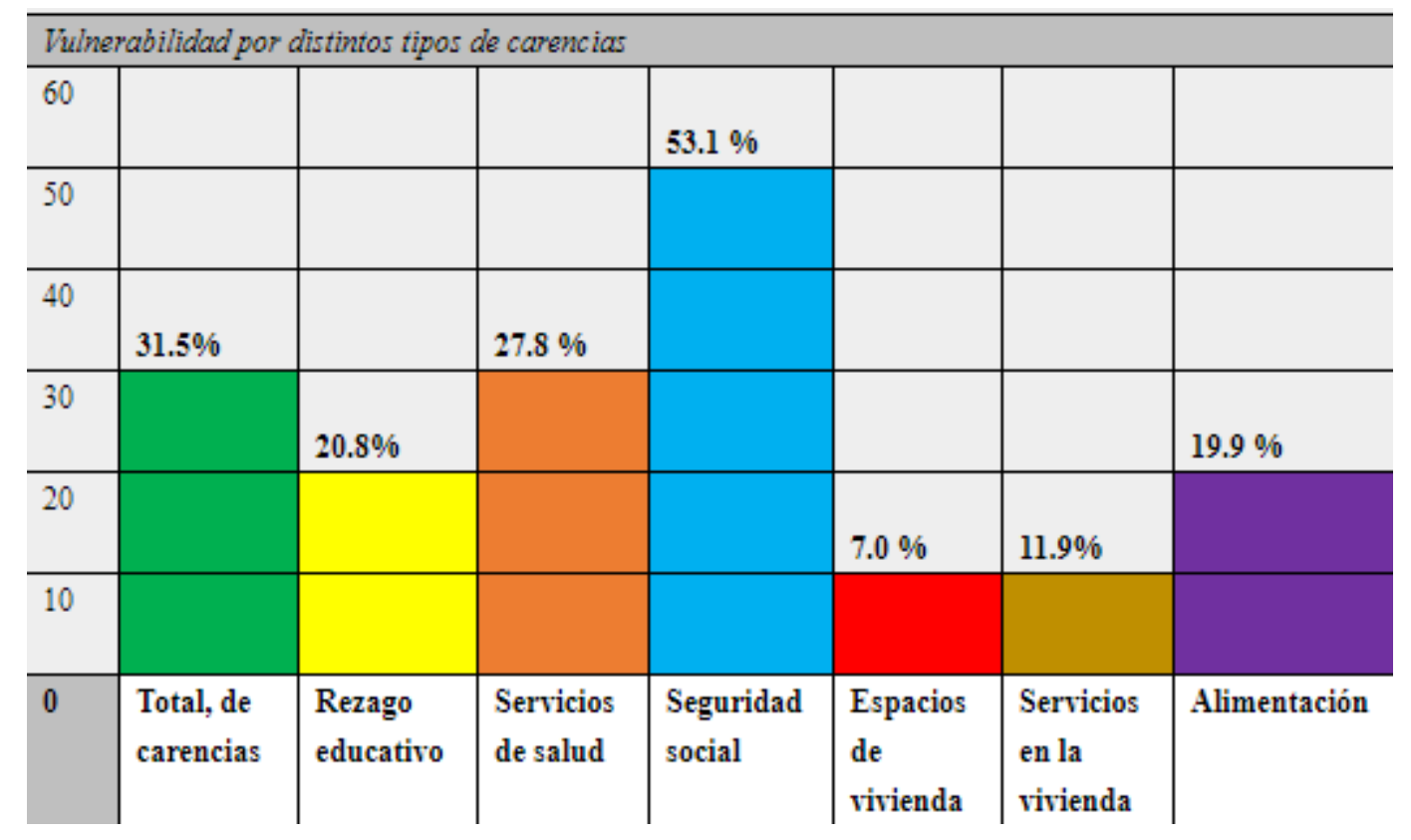

Figura 1. Análisis de la vulnerabilidad en la ciudad de León, Guanajuato. Fuente: Elaboración propia con base en datos del CONEVAL (2014).

2. Delimitación territorial-espacial. Desde una perspectiva territorial se analizan las distintas formas de concentración de la vulnerabilidad vinculadas estrechamente con la existencia de sectores urbanos desfavorecidos en distintas escalas territoriales.

En el caso de León, Guanajuato, se pudieron delimitar siete polígonos en estado de vulnerabilidad latente a través de contrastar indicadores relacionados con la pobreza y el rezago social, donde el número de personas que se encuentran en situación de pobreza en el municipio de León fue de 600 145, es decir, el equivalente al 37.8\%, de los que 533458 mil se encuentran en situación de pobreza moderada, es decir, el $33.6 \%$, y 66680 se encuentran en situación de pobreza extrema, es decir, el $4.2 \%$ de la población (OCL, 2012).

En la perspectiva de realizar una evaluación y análisis de los siete polígonos; se partió de la conceptualización y al cálculo de las variables que propone CONEVAL (2014), que son las siguientes:

- Ingreso corriente per cápita.

- Rezago educativo promedio en el hogar.

- Acceso a servicios de salud.

- Acceso a la seguridad social.

- Calidad y espacios en la vivienda.

- Acceso a servicios básicos en la vivienda.

- Acceso a la alimentación.

- Grado de cohesión social.

Al realizar el ejercicio de delimitar los polígonos en estado de vulnerabilidad utilizando el Marco Geo-estadístico del Instituto Nacional de Estadística y Geografía (INEGI 2000 y 2010), nos 
encontramos con tres diferentes casos en los que se encuentran los AGEB 'S de cada polígono:

1. Un alto porcentaje de las AGEB'S son comparables, ya que existen en los años 2000 y 2010.

2. Otras AGEB'S solo existen en el año 2000, por lo tanto, no son comparables con otro periodo, ya que no se generó el dato.

3. Otras AGEB'S solo existen en el año 2010 y actualmente no son comparables, pero seguramente lo serán con los resultados del Censo de población 2010.

Otro de los criterios que se propone tomar en cuenta para definir el status de los polígonos en vulnerabilidad, es tomar la correlación de las variables existentes de la información del año 2000 y 2010; al realizar el ejercicio los resultados nos mostraron una alta correlación entre grado de rezago educativo, carencias sociales e ingreso de la población.

Tomando como base este criterio, se propuso que los polígonos se clasifiquen en tres rangos (figura 2):

1. Polígonos que registran un crecimiento en el periodo 2000-2010. En los polígonos con este status, asumimos que las condiciones de marginación y pobreza además de seguir presentes, es un indicio de que las condiciones generales de la población continúan deteriorándose.

2. Poligonos que registran un comportamiento estable o moderado en el periodo 2000-2010. En este rango se encuentran los polígonos donde las condiciones de pobreza no han variado sustancialmente y continúan siendo socialmente focos rojos.

3. Poligonos que registran una disminución en el periodo 2000-2010. La evidencia de una disminución del rezago educativo y el nivel de ingresos, son un claro indicio de que los niveles de vida de la población tienen una tendencia positiva.

En conclusión, la vulnerabilidad urbana se expresa en: Baja calidad de vida, inseguridad en la vivienda y falta de servicios básicos, lo que comporta riesgos debidos a situaciones sanitarias críticas, contaminación y violencia. Riesgos debidos a la inestabilidad o inadecuada provisión de bienes básicos, incluyendo los sociales, humanos, financieros, físicos y naturales.

Discriminación y limitado acceso al mercado laboral, ingresos económicos inadecuados o inestables; traduciéndose también en la pérdida de los valores familiares y sociales tradicionales. 


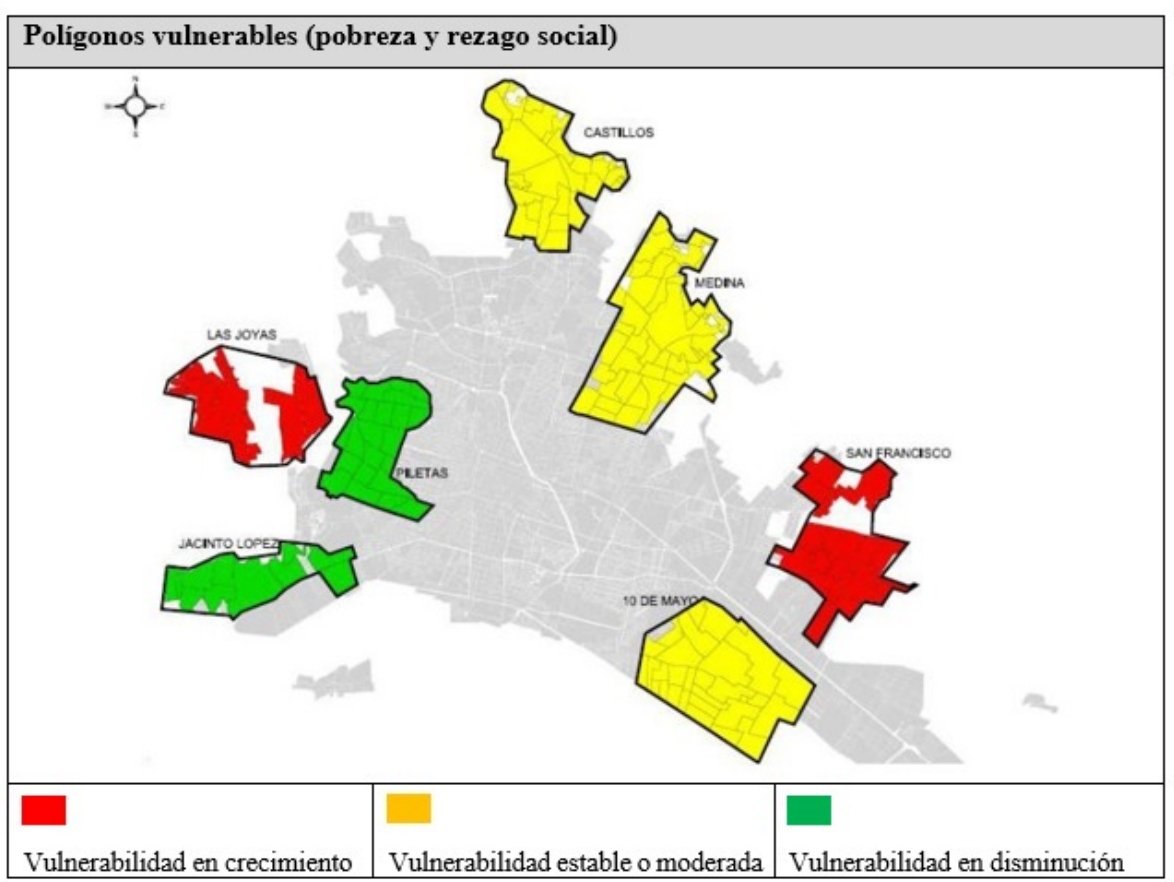

Figura 2. Identificación de polígonos vulnerables en la ciudad de León, Gto.

Fuente: Elaboración propia con base en el análisis de indicadores con información del INEGI 2000 y 2010

\section{Tipificación socio-espacial de la vulnerabilidad urbana}

A través de un recorrido por los polígonos vulnerables identificados y tras una caracterización urbano-arquitectónica por medio del levantamiento de información con base en fichas de análisis se propone una tipificación socio-espacial, dando como resultado las siguientes áreas:

- Áreas de atención especial

Áreas que sufren problemas urbanísticos y sociales que, en ocasiones, lejos de resolverse, tienden a agravarse con el tiempo. Entre dichos espacios destacan algunos donde se concentran procesos de deterioro urbano y riesgo ambiental dadas las características del territorio.

\section{Polígono de Los Castillos}

Ubicado en la zona norte de la ciudad, el polígono de Los Castillos presenta un incremento poblacional del $20 \%$, lo cual se tradujo en nuevos asentamientos irregulares en la zona, y en viviendas precarias, las cuales carecen de alguno de los servicios básicos, además de que la mayor parte de la zona no cuenta con pavimentación e infraestructura urbana.

Una de las características territoriales del polígono de Los Castillos, que dificultan el asentamiento de viviendas y urbanización, es lo accidentado del terreno, hecho que también contribuye a que muchas colonias del polígono sean de difícil acceso, de riesgo ambiental y en muchos sentidos excluidas del desarrollo urbano. Este polígono está constituido por 58 
colonias en las que se asentaron más de 64 mil personas según el conteo del año 2010.

- Áreas sensibles

Áreas que se han consolidado en infraestructura y equipamiento, pero enfrentan problemáticas de tipo económico y social, lo que ocasiona el deterioro físico del entorno.

\section{Polígono de Piletas}

De los siete polígonos identificados, Piletas además de ser el más urbanizado, también es el que presenta mejores condiciones para acceder a los servicios básicos, no solo a nivel de vivienda, sino en términos de la infraestructura y el equipamiento de la zona.

Desde el año 2000 se han mantenido sus 29 colonias, en términos demográficos, el comportamiento de la población en los años de referencia es muy estable dado que sólo se ha incrementado el $1 \%$ de su población en diez años, dicho polígono ha sido absorbido totalmente dentro de la mancha urbana.

\section{Polígono 10 de Mayo}

Es uno de los polígonos que se consolidó en términos urbanos, ya que se asentaron 10 colonias más, para pasar de 45 en el año 2000 a 55 en el 2010. Siendo éste, el polígono con mayor incremento de población, ya que su población se incrementó en 35734 habitantes, cifra que representa un $37.7 \%$ más de la población del año 2000.

En términos urbanos, el polígono 10 de Mayo registró una consolidación urbana importante, ya que sus colonias y lotes se densificaron. El polígono cuenta con una importante problemática juvenil, expresada en la proliferación de pandillas.

- Áreas en deterioro, degradación o declive

Proceso que se está dando en un área determinada y que tiene una clara dirección negativa en cuanto a falta de consolidación urbana en dos vertientes:

a) Se presentan situaciones de abandono del territorio ante la falta de dinámicas económicas y sociales.

\section{Polígono Jacinto López}

El polígono de Jacinto López está ubicado en un área suburbana, posiblemente de todos los polígonos es la zona que presenta mayor marginación y segregación urbana, ya que incluso su acceso es problemático. En el periodo de referencia (2000-2010), prácticamente la 
población no aumenta, pese a que existe suficiente superficie para que se desarrollen nuevos asentamientos.

En los años observados, las colonias registradas en la zona muestran una ligera disminución (de 17 a 15 colonias). Incluso se puede detectar en la zona un proceso de migración hacia otros sectores de la ciudad, ya que con el paso del tiempo no mejoran las condiciones generales de infraestructura y equipamiento urbano del polígono.

La irregularidad en la tenencia y el difícil acceso, posiblemente sean las dos principales causas que ha frenado la densificación poblacional de este asentamiento periférico.

b) Se presenta un crecimiento acelerado de población que se dispersa sobre el territorio e impide procesos de estabilidad y consolidación urbana.

\section{Polígono de Medina}

El polígono de Medina es la zona de pobreza más densamente poblada de los siete polígonos de la ciudad, para el 2010 registró 135240 habitantes. En cinco años (2005-2010) en el polígono se asentaron 10 colonias más para sumar 71 colonias (en cinco años se asentaron más de 35 mil habitantes). En la zona se localizan también asentamientos irregulares y los procesos y autoconstrucción tienen un fuerte impacto en el crecimiento de las viviendas.

En términos socio-demográficos, la población asentada en términos generales tiene características importantes de rezago educativo y de pobreza.

- Áreas desfavorecidas

Áreas donde la vulnerabilidad (ambiental, social, económica y política) materializa una situación de exclusión ya consolidada que dificulta la regeneración de determinadas áreas urbanas. Donde se presentan polígonos de viviendas o áreas de urbanización marginal realizadas sin una planificación ni dotación de equipamientos apropiados.

\section{Polígono de Las Joyas}

El polígono de Las Joyas, ubicado en la zona Nor-poniente de la ciudad, es una de las principales áreas de crecimiento de la población suburbana. $\mathrm{Su}$ estatus de carácter irregular representa una de las áreas con mayores carencias de equipamiento e infraestructura urbana de la ciudad.

El polígono de Las Joyas cuenta con todas las características socioeconómicas de un alto porcentaje de la población para ser considerado polígono de pobreza, su crecimiento poblacional se incrementó en el periodo observado en un $12.1 \%$, mientras que el nivel de analfabetismo aumentó el $16.4 \%$; mientras que en términos económicos 
un alto porcentaje de la población ocupada se ubica en ingresos que no superan los tres salarios mínimos mensuales.

\section{Polígono de San Francisco}

El polígono de San Francisco, ubicado al sur oriente de la ciudad, es una de las principales áreas de crecimiento y expansión de la mancha urbana. $\mathrm{Al}$ igual que la mayor parte de los suburbios de la ciudad, en ellos se entremezclan los asentamientos regulares e irregulares. En el periodo del 2000 al 2010 la población se incrementó en un 44.6 \% . Esta explosión poblacional se traduce en la autoconstrucción acelerada de vivienda.

El polígono de San Francisco incrementó su población en cinco años en 6250 habitantes. Los asentamientos humanos de la zona aún no se han consolidado territorialmente, por lo que se presentan nuevas colonias con déficits en servicios y equipamiento urbano.

\section{CONCLUSIONES}

\section{Aun cuando existe una gran variedad de aproximaciones teóricas para identificar \\ QUÉ HACE VULNERABLE A UN INDIVIDUO, \\ EXISTE UN CONSENSO CADA VEZ MÁS AMPLIO}

SOBRE LA NATURALEZA MULTIDIMENSIONAL DE ESTE CONCEPTO.

La vulnerabilidad, en su acepción más amplia, está asociada a condiciones de vida que vulneran la dignidad de las personas, limitan sus derechos y libertades fundamentales, impiden la satisfacción de sus necesidades básicas e imposibilitan su plena integración social.

Aun cuando existe una gran variedad de aproximaciones teóricas para identificar qué hace vulnerable a un individuo, existe un consenso cada vez más amplio sobre la naturaleza multidimensional de este concepto.

En este sentido, la vulnerabilidad está asociada también a la imposibilidad de disfrutar diversos satisfactores esenciales, muchos de los cuales son provistos por el Estado (tales como acceso a servicios de saneamiento o la seguridad pública), o que son considerados fundamentales por formar parte de los derechos humanos, económicos, sociales y culturales, lo cual involucra el análisis de distintas condiciones e indicadores de tipo social, económico, residencial, etc., por lo que en el trabajo realizado se propuso agrupar dichas condiciones en tres tipos de vulnerabilidad, la socio-demográfica, la socio-económica y la socioespacial que permitiera delimitar territorialmente áreas vulnerables.

Conscientes de que la complejidad de la problemática de la vulnerabilidad urbana requiere un análisis con mayor profundidad de cada una de las dimensiones consideradas (incorporando aspectos como la calidad de los servicios recibidos o el uso efectivo de los mismos), así como de otros aspectos que inciden directamente en el nivel de vida de la población (tales como el acceso a infraestructura o la seguridad ambiental) se realizó una tipificación socio-espacial con la finalidad de plantear un 
marco de investigación base para la implementación de estrategias de intervención, tanto en los ámbitos sociales como urbano-arquitectónicos.

Entender la vulnerabilidad urbana y su reflejo en el territorio, contribuirá a tomar medidas que busquen mejorar la capacidad de los seres humanos en estos sectores para afrontar y adaptarse positivamente a situaciones adversas.

\section{FUENTES DE CONSULTA}

Arriba González, A. (2008), Políticas y bienes sociales. Procesos de vulnerabilidad y exclusión social, Fundación FOESA (Fomento de Estudios Sociales y de Sociología Aplicada), Madrid.

Boltvinik, J., Hernández Laos, E. (1999), "Conceptos y Medidas de Pobreza”. En Pobreza y Distribución del Ingreso en México, Siglo XXI Editores, México.

Consejo Nacional de Evaluación de la Política de Desarrollo Social (CONEVAL) (2010), Índice de Rezago Social 2010, Consejo Nacional de Evaluación de la Política de Desarrollo Social, México.

Consejo Nacional de Evaluación de la Política de Desarrollo Social (CONEVAL) (2014), Informe de Pobreza 2014, Consejo Nacional de Evaluación de la Política de Desarrollo Social, México.

Consejo Nacional de Población (CONAPO) (2015), Índice de Marginación Urbana 2015, Consejo Nacional de Población, México.

Corredor Martínez, C. (2008), "Pobreza, equidad y eficiencia social". En Hábitat y desarrollo humano. Cuadernos PNUD-UN Hábitat, Colombia.

Cortez, F. (2002), "Consideraciones sobre la marginalidad, marginación, pobreza y desigualdad en la distribución del ingreso", Papeles de Población, vol. 8, núm. 31, enero-marzo, pp. 9-24.

Delgado, J. \& Galindo, C. (2007), “Índice de Consolidación Urbano-Regional”. En A. Coll Hurtado (coord.), Nuevo atlas nacional de México (Cap. Sociedad. Tema: Aglomeraciones urbanas multiescala, S XIII-1), Instituto de Geografía, UNAM, México.

Escuela Técnica Superior de Arquitectura de Madrid (ETSAM) (2014), Índice de desarrollo social en las ciudades, Escuela Técnica Superior de Arquitectura de Madrid, Madrid.

Feres, J. C., Mancero, X. (2001), El método de las Necesidades Básicas Insatisfechas (NBI) y sus aplicaciones en América Latina. División de Estadística y Proyecciones Económicas de la CEPAL, Santiago de Chile.

Instituto Nacional de Estadística y Geografía (INEGI) (2000), Censo de Población y Vivienda 2000, Instituto Nacional de Estadística y Geografía, México.

Instituto Nacional de Estadística y Geografía (INEGI) (2010), Censo de Población y Vivienda 2010, Instituto Nacional de Estadística y Geografía, México.

Ministerio de Fomento en España (MFE) (2010), Análisis urbanístico de Barrios Vulnerables en España, Instituto Juan de Herrera, Madrid.

Organización de las Naciones Unidas (ONU), Departamento de Asuntos Económicos y Sociales (DESA) (2003), Informe sobre la situación social 
del mundo 2003. Vulnerabilidad social: Fuentes y desafíos, United Nations Publications, Nueva York.

Observatorio Ciudadano de León (OCL) (2012), Análisis de Indicadores SIGU Sistema Integral de Gobernanza Urbana, Observatorio Ciudadano de León, México.

Palacio-Prieto, L., Sánchez-Salazar, M.T., Casado Izquierdo, J.M., Propin Frejomil, E., Delgado Campos, J., Velázquez Montes, A., Chias Becerril, L., Ortiz Álvarez, M.I., González Sánchez, J., Negrete Fernández, G., Gabriel Morales, J., Márquez Huitzil, R. (2004), Indicadores para la caracterización y el ordenamiento del territorio, Secretaría de Desarrollo Social, Secretaría de Medio Ambiente y Recursos Naturales, Instituto Nacional de Ecología, Universidad Nacional Autónoma de México, México.

Pizarro, R. (2001), La vulnerabilidad social y sus desafíos: una mirada desde América Latina. Serie Estudios Estadísticos y Prospectivos 6, Comisión Económica para América Latina (CEPAL), Santiago de Chile.

Programa de las Naciones Unidas para el Desarrollo (PNUD) (2016), Informe sobre Desarrollo Humano 2016, Programa de las Naciones Unidas para el Desarrollo, Nueva York.

Rodríguez, J. (2001), Vulnerabilidad y grupos vulnerables: un marco de referencia conceptual. Serie población y desarrollo 17, Comisión Económica para América Latina (CEPAL), Santiago de Chile.

Suárez, M., Ruiz, N., Delgado, J. (2012), "Desigualdad, desarrollo humano y la consolidación urbano-regional en México”, Revista EURE, vol. 38, núm. 115, septiembre, pp. 73-93. 